\title{
The electronic structure of pyrazine. Configuration interaction calculations using an extended basis*
}

\author{
W. R. Wadt, ${ }^{\dagger}$ W. A. Goddard III, ${ }^{\ddagger}$ and T. H. Dunning, Jr. ${ }^{\S}$ \\ Theoretical Division, Los Alamos Scientific Laboratory, Los Alamos, New Mexico 87544 \\ (Received 6 November 1975)
}

\begin{abstract}
Extensive $a b$ initio double zeta basis set configuration interaction calculations have been carried out on the $n \pi^{*}$ and $\pi \pi^{*}$ states of pyrazine as well as on the low-lying $n$ and $\pi$ cations. The calculations corroborate the validity of the valence bond (VB) model for the interaction of lone pair excitations proposed earlier by Wadt and Goddard. Good agreement (errors of $\sim 0.2 \mathrm{eV}$ ) with experiment is obtained (except for the higher-lying ${ }^{1} \pi \pi^{*}$ states that possess significant ionic character). The calculations indicate that the order of increasing ionization potentials is ${ }^{2} A_{g}(n), 2 B_{1 g}(\pi),{ }^{2} B_{1 u}(n)$, and ${ }^{2} B_{2 g}(\pi)$. The forbidden $1{ }^{1} B_{2 g}\left(n \pi^{*}\right)$ state is predicted to be $1.30 \mathrm{eV}$ above the allowed $1{ }^{1} B_{3 u}\left(n \pi^{*}\right)$ state. Finally, the calculations indicate that the adiabatic excitation energies to the $1^{3} B_{1}\left(n \pi^{*}\right)$ and $1^{3} A_{1}\left(\pi \pi^{*}\right)$ states in pyridine should be nearly degenerate at $\sim 3.6 \mathrm{eV}$
\end{abstract}

\section{INTRODUCTION}

The electronic structure of the low-lying states of pyrazine has provided a very fruitful area of research for spectroscopists and theoreticians alike. Not only are the benzenelike $\pi \rightarrow \pi^{*}$ transitions perturbed by the presence of the nitrogens, but they are also interspersed with new $n \rightarrow \pi^{*}$ transitions arising from the nonbonding (or lone pair) orbitals on the nitrogen. Moreover, the presence of two nitrogens in pyrazine leads to interactions between the $n \pi^{*}$ states arising from excitation out of the two different nonbonding orbitals.

Recently we proposed a model for describing the interaction of the lone pair excitations in terms of simple valence bond (VB) ideas. ${ }^{1}$ Extensive configuration interaction (CI) calculations using a minimal basis set (MBS) were carried out to test the VB model. The results show that the VB model provides a more accurate picture of the $n \pi^{*}$ states (and the $n$ cations) than the molecular orbital (MO) model. ${ }^{2}$

The MBS CI calculations led to good agreement with experimental exictation energies $( \pm 0.2 \mathrm{eV})$ for the lowest $^{1,3} n \pi^{*}$ states and the two $n$ cations. ${ }^{1}$ However, the calculated excitation energies to many $\pi \pi^{*}$ states, the cations, and the lowest Rydberg states were high by $0.5-2.5 \mathrm{eV} .{ }^{1,3}$ The high energies of the $\pi$ cations were clearly caused by the inflexibility of the MBS. The atomic scaling of the MBS led to $\sigma$ orbitals that were too diffuse, so that the $\pi$ orbitals were not properly shielded by the $\sigma$ core. On the other hand, some of the $\pi \pi^{*}$ states are known to have significant ionic character from CI studies on benzene. ${ }^{4}$ Thus, these states involve orbitals much more diffuse than the normal valence orbitals. Consequently, calculations employing a more flexible double zeta (DZ) basis were warranted. The results of extensive $\mathrm{DZ} C \mathrm{CI}$ calculations on the low-lying $n \pi^{*}, \pi \pi^{*}$, and cationic states of pyrazine are reported in this paper.

A brief review of the VB model is presented in the next section for purposes of interpreting the results of the calculations.

\section{SUMMARY OF THE VB MODEL}

In the VB framework, the nitrogen lone pairs in the ground state of pyrazine are assumed to be localized and equivalent, as shown in Diagrams $1 \mathrm{a}$ and $1 \mathrm{~b},{ }^{5,6}$
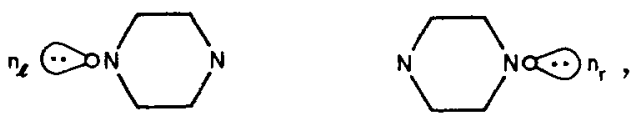

where the nonbonding orbitals are represented by $\propto$ and the electrons by dots. An $n$ cation is formed by ionizing an electron from either the left or right lone pair. The resulting equivalent configurations are combined (resonance) to form two $n$ cation states, ${ }^{2} A_{\varepsilon}$ and ${ }^{2} B_{1 u}$

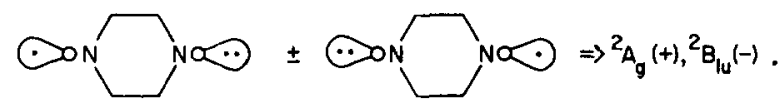

The splitting energy $\Delta E$ of the $n$ cations is proportional to the overlap $S_{n_{\imath} n_{r}}$ of the nonbonding orbitals, ${ }^{1}$ i. e.,

$$
\Delta E=E\left({ }^{2} A_{\varepsilon}\right)-E\left({ }^{2} B_{1 u}\right) \propto S_{n_{1} n_{r}} .
$$

Since the nitrogens are well separated (2.79 $\AA$ ), one would expect the overlap of the nonbonding orbitals to be small and positive, leading to the ${ }^{2} B_{1 u}$ state slightly below the ${ }^{2} A_{r}$. (In fact, the overlap of the atomic $s p$ hybrid nonbonding orbitals is 0.016 .) However, the effect on the atomic $n$ orbitals induced by the presence of the $\sigma$ and $\pi$ electrons in pyrazine must be taken into account.

Upon solving for the optimal wavefunctions [designated as generalized valence bond (GVB)] of the form shown schematically in Diagram 2, the most important effect is that induced by the Pauli principle. Because of the Pauli principle, there is a repulsive interaction between the nonbonding orbitals and the $\sigma$ and $\pi$ bonding orbitals. Therefore, for the optimal wavefunction, the nonbonding orbitals (singly - and doubly-occupied) build in nodal planes as shown in Diagram 3 in order to become orthogonal (Pauli principle) to the $\sigma_{\mathrm{CC}}$ and $\sigma_{\mathrm{CN}}$ bonding pairs:
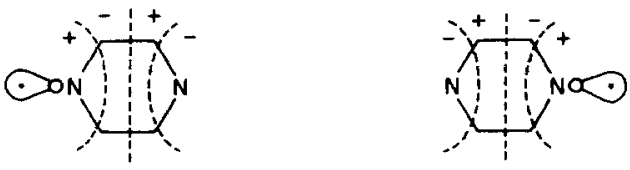

The resulting delocalization of the $n$ orbitals is slight, but the number and position of the nodal planes (cf. Diagram 3) lead to a significant decrease in $S_{n n_{r}}$. In 
fact, simple orthogonalization of atomic nonbonding orbitals to a symmetric $\sigma$ core produces nonbonding orbitals with an overlap of -0.106 . Using (1), the Pauliinduced orthogonalization of the nonbonding orbitals leads to a reversal in the ordering of the $n$ cation states $\left({ }^{2} A_{g}\right.$ below $\left.{ }^{2} B_{1 u}\right)$ as well as a large increase in the expected splitting energy.

For the $n \pi^{*}$ excited states we must consider the $\pi$ electrons explicitly. In the ground state the $\pi$ electrons may be coupled into the two VB Kekulé structures
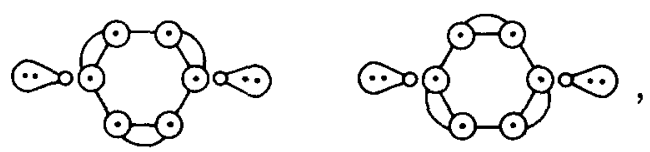

where the singlet-paired $\pi$ orbitals are connected by tie lines. In the VB model, the $n-\pi^{*}$ excitation involves the promotion of an electron from the nonbonding orbital to the $p_{\text {r }}$ orbital on the same nitrogen. The five singly occupied $p_{r}$ orbitals are coupled into $\pi$ bonds, leading to

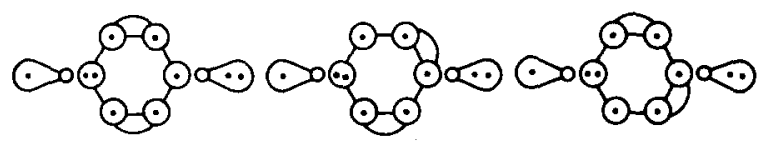

as the dominant terms in the wavefunction. Thus, the resulting unpaired $p$, orbital (the $\pi^{*}$ orbital) is on either the ortho carbons or para nitrogen, even though the electron promotion is localized on a single nitrogen. Combining (resonance) the configurations in Diagrams 5 with a comparable set obtained by exciting the right nitrogen gives $B_{3 u}$ and $B_{2 g}$ states

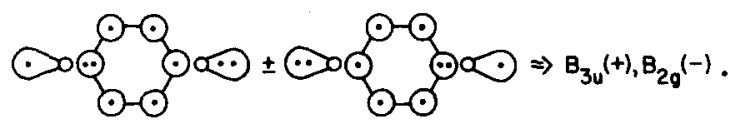

The splitting of the $n \pi^{*}$ states may be shown to be proportional to the product of the nonbonding and $\pi^{*}$ orbital overlaps, 1

$$
\Delta E=E\left(B_{3 u}\right)-E\left(B_{2 g}\right) \propto S_{n_{i} n_{r}} S_{r_{i}^{*} r_{r}^{*}} .
$$

Since the $\pi^{*}$ orbitals are quite delocalized, the splitting energy of the $n \pi^{*}$ states is expected to be only slightly smaller than that of the $n$ cations. Moreover, the overlap of the $\pi^{*}$ orbitals may be shown to be positive ${ }^{1}$ so that $\Delta E$ is negative and the $B_{3 u}$ states are expected to be below the $B_{2 r}$ states.

Finally, the unpaired nonbonding and $\pi^{*}$ orbitals may be coupled into a singlet or a triplet, the energies of which are given by

$$
\begin{aligned}
& E\left({ }^{\mathrm{l}} n \pi^{*}\right)=E_{0}+K, \\
& E\left({ }^{3} n \pi^{*}\right)=E_{0}-K,
\end{aligned}
$$

where $K=\left\langle n \pi^{*} \mid n \pi^{*}\right\rangle$ is the two-electron exchange integral between the unpaired $n$ and $\pi^{*}$ orbitals. Since $K$ is positive, the triplet state is lower than the singlet. Increasing the size of $K$ lowers the energy of the ${ }^{3} n \pi^{*}$ state, while decreasing the size of $K$ lowers the energy of the ${ }^{1} n \pi^{*}$ state. Therefore, configurations $5 \mathrm{~b}$ and $5 \mathrm{c}$ with the $\pi^{*}$ orbital on the ortho carbon are favored for the triplet, while 5a with the $\pi^{*}$ orbital on the para nitrogen is favored for the singlet. In fact, to increase further the size of $K$ for the ${ }^{3} n \pi^{*}$ state, the following ionic configurations are expected to contribute significantly to the VB wavefunction:

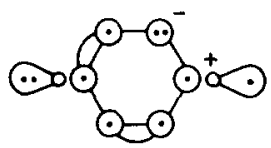

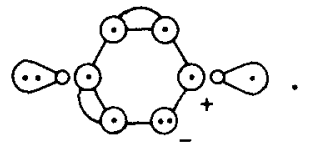

\section{CALCULATIONAL DETAILS}

\section{A. Basis set and geometry}

As before, ${ }^{1}$ all calculations were performed using the experimental geometry determined by Wheatley, ${ }^{7}$ except that the $\mathrm{CH}$ bond lengths were taken to be 1.09 $\AA$ instead of $1.05 \AA$, in line with the electron diffraction studies. ${ }^{8}$ The basis set employed for all the calculations was Dunning' $s^{9,10}$ double zeta quality $[3 s, 2 p /$ $2 s$ ] contraction of Huzinaga' ${ }^{11}(9 s, 5 p / 4 s)$ set of primitive Gaussian basis functions (the $3 s$ set has one $1 s$-like function and two $2 s$-like functions).

\section{B. Wavefunctions}

As discussed previously, ${ }^{1}$ the most feasible way to describe properly the $n$ cations and $n \pi *$ states, which involve the interaction of two $n$ equivalent excitations, is by CI calculations. The procedures for constructing good quality CI wavefunctions developed in the MBS calculations ${ }^{1}$ will be used without significant alteration. The specifics are outlined below.

\section{Localization of the $n$ orbitals}

All the CI calculations on the neutral excited states are carried out using the ground state Hartree-Fock (HF) orbitals, while the appropriate HF cation orbitals are used for the CI calculations on the $n$ and $\pi$ cations. To facilitate the CI calculations, the occupied orbital space is partitioned into $n$ and $\sigma$ orbitals ${ }^{12}$ by performing open-shell HF calculations with a fully convergent SCF program ${ }^{13}$ on the ${ }^{2} n,{ }^{1} n \pi^{*}$, and ${ }^{3} n \pi^{*}$ states, allowing the symmetry to be reduced to $C_{2 v}$. The singly occupied nonbonding orbital is uniquely defined in each of the three wavefunctions. As shown by the contour plots in Fig. 1 and the Mulliken populations in Table I,

\begin{tabular}{|c|c|c|c|c|c|}
\hline & & & ${ }^{2} n$ & ${ }^{1} n \pi^{*}$ & ${ }^{3} n \pi^{*}$ \\
\hline \multirow{3}{*}{ Excited } & \multirow{3}{*}{$\mathrm{N}$} & $s$ & 0.162 & 0.141 & 0.163 \\
\hline & & $z$ & 0.712 & 0.730 & 0.717 \\
\hline & & Total & 0.874 & 0.871 & 0.880 \\
\hline ortho & $\mathrm{C}$ & & 0.027 & 0.029 & 0.026 \\
\hline ortho & $\mathrm{H}$ & & 0.009 & 0.008 & 0.008 \\
\hline meta & C & & 0.021 & 0.021 & 0.020 \\
\hline meta & $\mathrm{H}$ & & 0.003 & 0.004 & 0.003 \\
\hline para & $\mathbf{N}$ & & 0.007 & 0.005 & 0.006 \\
\hline
\end{tabular}

TABLE I. Mulliken populations for the singly occupied $n$ orbital. 
the singly-occupied non-bonding orbital
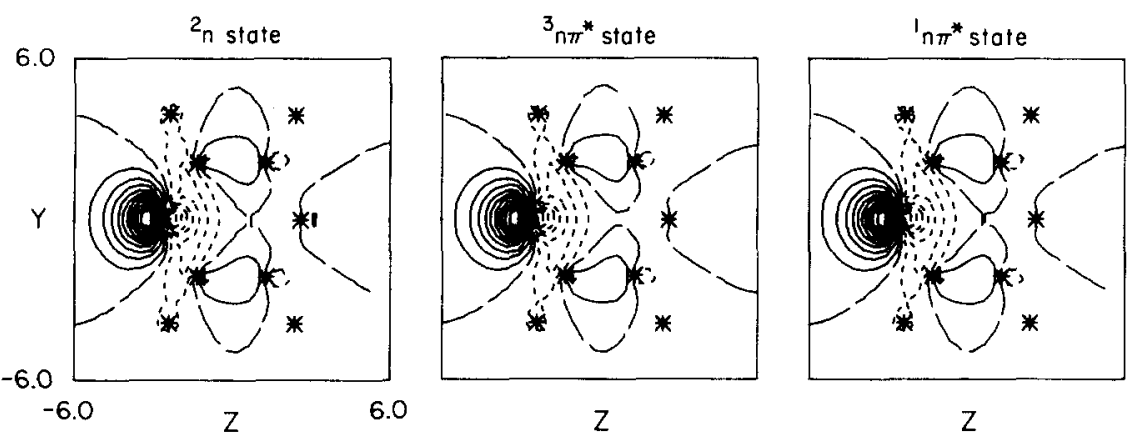

FIG. 1. Contour plots of the singly occupied $n$ orbital from the ${ }^{2} n,{ }^{3} n \pi^{*}$, and ${ }^{1} n \pi^{*} \mathrm{SCF}$ wavefunctions. The contour increment is $0.05 \mathrm{a} . \mathrm{u}$.

the nonbonding orbital is quite localized on one nitrogen $(\sim 88 \%)$, just as in the MBS calculations. ${ }^{1}$ Since all three nonbonding orbitals are so similar, we chose one, the ${ }^{3} n \pi^{*}$ orbital, to partition the HF space into $n$ and $\sigma$ orbitals.

Taking the localized $n$ orbital, symmetry functions, $n_{+}$and $n_{-}$, were formed and projected onto the doubly occupied HF orbitals for the ${ }^{1} A_{g},{ }^{2} A_{s},{ }^{2} B_{1 u},{ }^{2} B_{1 g}$, and ${ }^{2} B_{2 \varepsilon}$ states. The remaining $\sigma$ orbitals were orthogonalized to the $n$ orbitals. In this manner, the HF-occupied space was partitioned into $n$ and $\sigma$ orbitals.

\section{Partitioning of virtual $\pi$ space}

In the MBS calculations, we performed full CI calculations among the two $n$ and six $\pi$ orbitals, finding that the three unoccupied ground state $\pi$ orbitals are very important (and effective) for correlating the $\pi$ electrons, leading to a CI energy lowering of $2.49 \mathrm{eV}$ in the ground state. ${ }^{1}$ In order to minimize the number of configurations required for the $\mathrm{DZ}$ CI calculations, it is necessary to extract from the set of nine virtual $\pi$ orbitals the three that would most effectively correlate the $\pi$ electrons. Assuming that the appropriate functions would be similar in character to the MBS $\pi$ virtuals, we took a set of atomic-scaled DZ $p_{x}$ functions on each carbon and nitrogen, constructed the three symmetry functions with two or three nodes (apart from the node in the molecular plane), and orthogonalized them to the occupied HF $\pi$ orbitals for the ground state or appropriate cation. The effectiveness of this procedure is borne out by a full $\pi \mathrm{CI}$ on the ground state, which led to a CI energy lowering of $2.34 \mathrm{eV}$, comparable to that of the MBS calculation. The remaining six DZ $\pi$ virtuals were orthogonalized to the six "MBS" $\pi$ orbitals.

In one set of $\mathrm{CI}$ calculations [MBS $(\sigma)$ POL (1) CI] we have included 20 additional functions of $\sigma$ symmetry. These included the ten molecular orbitals describing the $\mathrm{CH}, \mathrm{CN}$, and $\mathrm{CC}$ bonds plus ten virtual orbitals. The ten virtual functions were constructed by combining $\mathrm{DZ}$ atomic orbitals to mimic the $\sigma$ virtuals in the MBS calculations.

\section{Cl calculations}

Three types of CI calculations were performed.

a. GVB CI: A full CI among the two $n$ and six MBS $\pi$ orbitals for the $n$ cation and $n \pi^{*}$ states or a full CI among the six MBS $\pi$ orbitals for the $\pi$ cation and $\pi \pi^{*}$ states.

b. $D Z(\pi) P O L(3) C I$ : GVB CI plus all the configurations that arise from triple excitations from the ground state HF configuration [within a 14 orbital ( $2 n, 6$ MBS $\pi$ $6 \mathrm{DZ} \pi$ ) space $\left.{ }^{14}\right]$ with the restriction that only single excitations are allowed into the set of $\operatorname{six~DZ} \pi$ virtual functions. ${ }^{15}$

c. $M B S(\sigma) P O L(1) C I$ : GVB CI plus all the configurations that arise from single excitations from the set of parent configurations [within a 28 orbital (2n, 20 MBS $\sigma, 6 \mathrm{MBS} \pi$ ) space] for each state. The parent configurations consisted primarily of the dominant configurations in the GVB CI wavefunction for the low-lying states of each symmetry and were generated as follows. For the ground state and $\pi \pi^{*}$ states (symmetries $A_{\varepsilon}, B_{1 u}$, $\left.B_{2 u}, B_{3 \xi}\right)$, the parent configurations consisted of the dominant configurations for the lowest state of each symmetry (one for $A_{g}$; two for the others) plus all single excitations within the MBS $\pi$ space that retain the state symmetry. The parent configurations for ${ }^{1} A_{8}$ and ${ }^{3} B_{2 u}$ were augmented with configurations (two and one, respectively) that describe the states corresponding to the double excitation $n_{1} n_{r}-\pi_{1} \pi_{r}$. The result is five parent configurations for ${ }^{1} A_{k}$, two for ${ }^{3} A_{k}$, seven for ${ }^{3} B_{1 u}$, and six for the remaining symmetries. For the $n \pi^{*}$ states (symmetries $B_{3 u}, B_{2 g}, A_{u}, B_{1 \xi}$ ), we included the dominant configuration for the lowest state of each symmetry plus the configuration that allows the lone pair excitation to localize. ${ }^{1,16}$ Then all single excitations from the localizing configuration that lead back to the symmetry of the dominant configuration are included. These configurations, which are double excitations relative to the dominant configuration, have the next largest coefficients in the GVB CI wavefunction. Finally, we include the localizing configuration for each of these double excitations. The result is eight parent configurations for each symmetry.

\section{RESULTS}

\section{A. $n$ and $\pi$ cations}

The DZ and MBS SCF results for the four lowest ionization potentials are compared in Table II. The flexible DZ basis leads to decreases of 1.2 and $2.0 \mathrm{eV}$ for the $n$ and $\pi$ ionization potentials, respectively, 
TABLE II. Comparison of MBS and DZ SCF results (in eV).

\begin{tabular}{lrr}
\hline \hline State & MBS & \multicolumn{1}{c}{ DZ } \\
\hline${ }^{1} A_{g}$ & $0.0^{2}$ & $0.0^{\mathrm{b}}$ \\
${ }^{3} n \pi^{*}$ & 3.41 & 3.63 \\
${ }^{1} n \pi^{*}$ & 4.09 & 4.25 \\
${ }^{2} n$ & 10.51 & 9.27 \\
${ }^{2} A_{g}(n)$ & 11.32 & 10.20 \\
${ }^{2} B_{11}(n)$ & 13.55 & 12.11 \\
${ }^{2} B_{1 g}(\pi)$ & 11.68 & 9.66 \\
${ }^{2} B_{2 g}(\pi)$ & $\cdots$ & 11.13 \\
\hline$=$
\end{tabular}

a 261.95270 a. u.

b-262. 58320 a. u.

which implies that the atomic scaling of the MBS was not appropriate for the cations. On the other hand, the effect of allowing the lone pair ionization to localize on one nitrogen is comparable in both basis sets. The resulting significant decrease in energy $(0.81$ and $0.93 \mathrm{eV}$ for the MBS and DZ bases, respectively) verifies the validity of the VB description of the $n$ cations as resonant and antiresonant combinations of localized ionizations.

The DZ and MBS CI results are shown in Table III. The GVB CI calculation allows the localized lone pair ionizations to interact, leading to $E\left({ }^{2} A_{g}\right)<E\left({ }^{2} B_{1 u}\right)$, as predicted by the VB model. The GVB CI also leads to an increase of $0.2-0.3 \mathrm{eV}$ in the $\pi$ ionization potentials, since the six-electron $\pi$ system of the ground state has more correlation energy than five-electron $\pi$ systems of the $\pi$ cations. The $\mathrm{DZ}(\pi)$ POL(3) CI has a negligible effect on the ionization potentials as we are employing the optimal SCF orbitals in each case. Finally, although $\operatorname{MBS}(\sigma) \operatorname{POL}(1) \mathrm{CI}$ calculations were not carried out on the cations, the results should be comparable to those obtained in MBS calculations. ${ }^{17}$ Therefore, we would expect the relaxation of the $\sigma$ system to have a negligible effect on the $\pi$ cations, while the energies of the $n$ cations would be lowered by $0.25-0.50 \mathrm{eV}$.

The DZ CI calculations predict the ordering of the pyrazine ionization potentials to be ${ }^{2} A_{g}(n),{ }^{2} B_{1 g}(\pi)$, ${ }^{2} B_{1 u}(n),{ }^{2} B_{2 g}(\pi)$. This result is in accord with our previous assignment made on the basis of the MBS CI results as well as with the assignment of Gleiter, Heilbronner, and Hornung ${ }^{18}$ based on photoelectron band shapes. Comparison of the DZ CI results with experi- ment reveals that the calculated $\pi$ ionization energies are in good agreement (0.1-0.3 eV errors), while the calculated $n$ ionization energies are too low by $\sim 1 \mathrm{eV}$ when relaxation of the $\sigma$ system is taken into account. The origin of the $1 \mathrm{eV}$ error in the $n$ ionization potentials is the correlation energy involving the lone pair. Extensive CI calculations on the low-lying $(n-R)$ Rydberg states of water indicate a correlation energy of $\sim 1.2$ $\mathrm{eV}$ for the oxygen lone pairs. ${ }^{19}$ Therefore, the size of the error in the $n$ ionization energies introduced by the $\mathrm{HF}$ description of the nitrogen lone pair is reasonable.

\section{B. $n \pi^{*}$ and $\pi \pi^{*}$ excited states}

The DZ SCF and CI results are listed in Table II and IV, respectively, along with the MBS results for comparison. In addition, the number of configurations and spin eigenfunctions as well as the energy lowering for a representative set of CI calculations are given in Table V. The DZ SCF ${ }^{1,3} n \pi^{*}$ excitation energies are slightly larger $(0.2 \mathrm{eV})$ than their MBS counterparts, indicating that the atomic scaling of the MBS is more appropriate for the $n \pi^{*}$ states than the ground state. Again the GVB CI calculation allows the two localized $n \rightarrow \pi$ excitations to interact. The resultant splitting is in agreement with the predictions of the VB model, i. e., $E\left({ }^{1,3} B_{3 u}\right)<E\left({ }^{1,3} B_{2 g}\right)$. In addition, the GVB CI leads to a general increase in the $n \pi^{*}$ excitation energies, since the correlation energy of the seven-electron $\pi$ system is less than that of the six-electron $\pi$ system within the limitations of a six-orbital (MBS) description. ${ }^{20}$

The DZ( $\pi$ ) POL(3) CI is equivalent to the GVB CI except that the six-orbital $\pi$ space is allowed to adjust for each excited state. (In the GVB CI the ground state $\pi$ orbitals are used in each case.) As expected, this relaxation leads to a large decrease in energy (0.6-0.8 $\mathrm{eV}$ ) for the $n \pi^{*}$ states with seven-electron $\pi$ systems and a smaller decrease $(0.1-0.2 \mathrm{eV})$ for the $\pi \pi^{*}$ states with six-electron $\pi$ systems. A large decrease $(0.9$ $\mathrm{eV})$ is also obtained for the double excitation $\left(n \rightarrow \pi^{*}\right.$, $\left.n \rightarrow \pi^{*}\right)$ states, $2{ }^{1} A_{g}$ and $3{ }^{3} B_{1 u}$, with eight-electron $\pi$ systems.

The MBS( $\sigma)$ POL(1) CI allows the ground state $\sigma$ orbitals to relax in response to the $n \rightarrow \pi^{*}$ or $\pi \rightarrow \pi^{*}$ excitation. The effect of the $\sigma$ relaxation is minimal for the $\pi \pi^{*}$ states, which indicates that the excitations are delocalized over the system. On the other hand, the

TABLE III. CI results for the vertical ionization potentials (in eV).

\begin{tabular}{|c|c|c|c|c|c|c|}
\hline \multirow[b]{2}{*}{ State } & \multicolumn{3}{|c|}{ MBS CI } & \multicolumn{2}{|c|}{$\mathrm{DZ} \mathrm{CI}$} & \multirow[b]{2}{*}{ Exptl } \\
\hline & GVB & $\mathrm{DZ}(\pi) \mathrm{POL}(2)$ & $\operatorname{MBS}\left(\sigma^{*}\right) \operatorname{POL}(1)^{2}$ & GVB & $\mathrm{DZ}(\pi) \mathrm{POL}(3)$ & \\
\hline $1^{2} A_{g}(n)$ & 10.27 & $\cdots$ & 9.81 & 9.13 & 9.12 & 9.63 \\
\hline$I^{2} B_{1 g}(\pi)$ & $12.48^{\mathrm{c}}$ & $12.29^{\mathrm{c}}$ & $12.34^{\mathrm{c}}$ & 10.01 & 10.08 & 10.18 \\
\hline $1^{2} B_{1 u}(n)$ & 11.70 & $\cdots$ & 11.45 & 10.48 & 10.48 & 11,35 \\
\hline $1^{2} B_{2 g}(\pi)$ & $13.36^{\mathrm{c}}$ & $13.16^{\mathrm{c}}$ & $13.25^{c}$ & 11.32 & 11.41 & 11.77 \\
\hline
\end{tabular}

${ }^{2}$ Excitations allowed only within $\sigma_{\mathrm{CC}}$ and $\sigma_{\mathrm{CN}}$ space, relaxation of $\sigma_{\mathrm{CH}}$ space is of secondary importance (cf. Ref. 1 for details).

${ }^{b}$ Reference 18 .

${ }^{c} \mathrm{Ground}$ state orbitals are used for the cation. 
TABLE IV. CI results for the vertical excitation energies (in eV).

\begin{tabular}{|c|c|c|c|c|c|c|c|c|c|}
\hline \multirow[b]{2}{*}{ State } & \multicolumn{4}{|c|}{ MBS CI } & \multicolumn{4}{|c|}{$\mathrm{IZZCI}$} & \multirow{2}{*}{$\begin{array}{l}\text { Experiment } \\
\text { V: Vertical } \\
\text { A: Adiabatic }\end{array}$} \\
\hline & GVB & $\mathrm{DZ}(\pi) \mathrm{POL}(3)$ & $\operatorname{MBS}\left(\sigma^{*}\right) \operatorname{POL}(1)^{2}$ & $\begin{array}{l}\text { Best } \\
\text { estimate }\end{array}$ & GVB & $\mathrm{DZ}(\pi)$ POL $(3)$ & $\operatorname{MBS}(\sigma) \mathrm{POL}(1)$ & $\begin{array}{l}\text { Best } \\
\text { estimate }\end{array}$ & \\
\hline $1^{1} A_{g}$ (G.S.) & $0.0^{\mathrm{b}}$ & $0.0^{\mathrm{b}}$ & $0.0^{\mathrm{b}}$ & 0.0 & $0.0^{\mathrm{b}}$ & $0.0^{\mathrm{b}}$ & $0.0^{\mathrm{b}}$ & 0.0 & \\
\hline $1{ }^{3} B_{3 u}\left(n \pi^{*}\right)$ & 3.45 & 3.10 & 2.94 & 2.59 & 4.36 & 3.78 & 3.86 & 3.28 & $3.2-3.3^{\mathrm{c}}(\mathrm{V})$ \\
\hline $1^{1} B_{3 u}(n \pi *)$ & 4.23 & 3.91 & 3.75 & 3.43 & 5.10 & 4.51 & 4.59 & 4.00 & $3.8-3.9^{\mathrm{c}}(\mathrm{V})$ \\
\hline $1^{3} B_{1 u}\left(\pi \pi^{*}\right)$ & 4.14 & 4.07 & $\ldots$ & 4.07 & 4. 38 & 4.11 & 4.49 & 4.11 & $3.4-3.5^{d}(\mathrm{~A})$ \\
\hline $1{ }^{3} B_{2 g}\left(n \pi^{*}\right)$ & 4.70 & 4.41 & 4.31 & 4.02 & 5.46 & 4.92 & 5.10 & 4.56 & $\sim 4.2^{e}(\mathrm{~A})$ \\
\hline $1^{3} A_{u}\left(n \pi^{*}\right)$ & 5.34 & 4.88 & $\cdots$ & 4.88 & 6.57 & 5.74 & 5.55 & 4.72 & $\sim 4.5^{\circ}(\mathrm{A})$ \\
\hline $1^{1} A_{u}\left(n \pi^{*}\right)$ & 5.37 & 4.90 & $\cdots$ & 4.90 & 6.60 & 5.76 & 5.55 & 4.72 & $\cdots$ \\
\hline $1{ }^{3} B_{2 u}\left(\pi \pi^{*}\right)$ & 5.59 & 5.36 & $\cdots$ & 5.36 & 5.66 & 5.09 & 5.52 & 5.09 & $\cdots$ \\
\hline $1^{1} B_{2 u}\left(\pi \pi^{*}\right)$ & 5.41 & 5.26 & $\cdots$ & 5.26 & 5.57 & 5.16 & 5.66 & 5.16 & $4.8-4.9^{\mathrm{c}}$ (V) \\
\hline $1^{1} B_{2 \pi}\left(n \pi^{*}\right)$ & 5.38 & 5.12 & 5.05 & 4.79 & 6.13 & 5.58 & 5.85 & 5.30 & $\sim 5.5^{2}(\mathrm{~A})$ \\
\hline $2{ }^{3} B_{1 u}\left(\pi \pi^{*}\right)$ & 5.58 & 5.41 & $\cdots$ & 5.41 & 5.83 & 5.36 & 5.97 & 5.36 & $\cdots$ \\
\hline $1{ }^{3} B_{1 g}\left(n \pi^{*}\right)$ & 6.68 & 6.27 & $\cdots$ & 6.27 & 7.75 & 6.95 & 7.03 & 6.23 & $\ldots$ \\
\hline $1^{1} B_{1 g}\left(n \pi^{*}\right)$ & 6.83 & 6.41 & $\cdots$ & 6.41 & 7.88 & 7.06 & 7.12 & 6.30 & $\cdots$ \\
\hline $2^{1} A_{g}\left(n \pi^{*}\right)^{?}$ & 6.97 & 6.54 & $\cdots$ & 6.54 & 8.69 & 7.81 & & $\cdots$ & $\cdots$ \\
\hline $3^{3} B_{1 u}^{8}\left(n \pi^{*}\right)^{p}$ & 7.33 & 7.41 & $\cdots$ & 7.41 & & & & $\cdots$ & $\cdots$ \\
\hline $1^{3} A_{R}\left(\pi \pi^{*}\right)$ & 7.80 & 7.67 & $\cdots$ & 7.67 & 7.99 & 7.60 & 8.63 & 7.60 & $\ldots$ \\
\hline $1^{3} B_{3 R^{*}}\left(\pi \pi^{*}\right)$ & 7.88 & 7.73 & $\cdots$ & 7.73 & 8.21 & 7.78 & 8.51 & 7.78 & $\cdots$ \\
\hline $2{ }^{3} B_{2 u}\left(\pi \pi^{*}\right)$ & 9.20 & 8.62 & $\cdots$ & 8.62 & 9.54 & 8.37 & 9.08 & 8.37 & $\cdots$ \\
\hline $2^{3} B_{2 p}\left(n \pi^{*}\right)$ & 8.33 & 7.93 & $\cdots$ & 7.93 & 9.48 & 8.63 & 9.38 & 8.53 & $\cdots$ \\
\hline $2{ }^{1} B_{2 g}\left(n \pi^{*}\right)$ & 8.47 & 8.09 & $\cdots$ & 8.09 & 9.61 & 8.80 & 9.48 & 8.67 & $\cdots$ \\
\hline $3^{1} A_{k}\left(\pi \pi^{*}\right)$ & 9.04 & 8.85 & $\cdots$ & 8.85 & 9.29 & 8.70 & 9.89 & 8.70 & $\cdots$ \\
\hline $1{ }^{1} B_{3 \boldsymbol{g}}\left(\pi \pi^{*}\right)$ & 9.04 & 8.90 & $\cdots$ & 8.90 & 9.40 & 8.81 & 9.75 & 8.81 & $\cdots$ \\
\hline $1^{1} B_{14}\left(\pi \pi^{*}\right)$ & 9.87 & 9.31 & $\ldots$ & 9.31 & 10.09 & 8.81 & 10.05 & 8.81 & $6.5-6.6^{\mathrm{c}}(\mathrm{V})$ \\
\hline $2{ }^{1} B_{3 u}\left(n \pi^{*}\right)$ & 9.44 & 9.11 & $\cdots$ & 9.11 & 10.45 & 9.66 & 10.50 & 9.71 & $\cdots$ \\
\hline $2{ }^{1} B_{2 u}\left(\pi \pi^{*}\right)$ & 10.93 & 10.36 & $\cdots$ & 10.36 & 11.16 & 9.80 & 10.93 & 9.80 & $7.6-7.7^{c}(\mathrm{~V})$ \\
\hline $2{ }^{1} B_{3 u}\left(n \pi^{*}\right)$ & 9.64 & 9.34 & $\cdots$ & 9.34 & 10.63 & 9.87 & 10.74 & 9.98 & $\cdots$ \\
\hline $2{ }^{1} B_{1 u}\left(\pi \pi^{*}\right)$ & $\cdots$ & $\cdots$ & $\cdots$ & $\cdots$ & 11.83 & 10.52 & 11.86 & 10.52 & $\cdots$ \\
\hline
\end{tabular}

${ }^{2}$ See Footnote a in Table III.

${ }^{b}$ The ground state energies (in a.u.) from left to right are $-262.04408(-262.04180),-262.05872(-262.05613),-262.03992$ $(-272.03754),-262.67107(-262.66930),-262.67916(-262.67725),-262.69588(-262.69391)$. The CI energies in which only the $\pi$ space was used are listed parenthetically.

${ }^{c}$ References 27 and 28 .

${ }^{\mathrm{d}}$ Reference 22 .

Reference 26 .

${ }^{\text {I }}$ Dominant configuration(s) involves a double excitation, $n \rightarrow \pi^{*}, n \rightarrow \pi^{*}$.

Reference 41.

MBS( $\sigma$ ) POL(1) CI leads to large (0.5-1.0 eV) decreases in the $n \pi^{*}$ excitation energies, which arise from the polarization of the $\sigma$ core in response to the localized $n-\pi^{*}$ excitations. Again the predictions of the VB model are borne out.

Since the configurations added to the GVB CI in the two POL CI's are mutually exclusive (exactly in a mathematical sense and roughly in a physical sense), one can sum the respective CI lowerings to get a reasonable estimate of the CI energies that would result if the POL CI's were carried out simultaneously. The results of combining the POL CI's are listed in Table $\mathrm{V}$ under the heading "Best Estimate." Since the effect of the $\operatorname{MBS}(\sigma) \operatorname{POL}(1) \mathrm{CI}$ on the $\pi \pi^{*}$ states is minimal, the $\mathrm{DZ}(\pi)$ POL(3) CI results are used for the best estimate.

\section{DISCUSSION}

In the following paragraphs we will compare our best estimates for the calculated vertical excitation energies with experiment in order of increasing energy. The theoretical and experimental results are listed in Table IV.

The calculated excitation energies of the $1^{1,3} B_{3 u}\left(n \pi^{*}\right)$ states are in good agreement with experiment $(\sim 0.1 \mathrm{eV}$ errors). These results may seem surprising in light of the $1 \mathrm{eV}$ errors encountered with the lone pair ionization potentials. However, in the $n \pi^{*}$ states the correlation energy of the nitrogen lone pair in the ground state is balanced by that of the doubly occupied nitrogen $p_{\mathbf{r}}$ orbital in the excited state (cf. Diagrams 1 and 5), i.e., there is no net change in the number of doubly occupied

TABLE V. Size of the CI calculations.

\begin{tabular}{|c|c|c|c|c|}
\hline State & & GVB & $\mathrm{DZ}(\pi)$ POL $(3)$ & $\operatorname{MBS}(\sigma) \operatorname{POL}(1)$ \\
\hline \multirow{3}{*}{${ }^{1} A_{\mathrm{z}}$} & Configurations & 128 & 324 & 273 \\
\hline & Spin eigenfunctions & 176 & 532 & 379 \\
\hline & $\Delta E^{2}$ & 0.0 & 0.22 & 0.68 \\
\hline \multirow{3}{*}{$\begin{array}{l}{ }^{1} A_{c} \\
(\pi \text { only })^{\mathrm{b}}\end{array}$} & Configurations & 42 & 139 & 129 \\
\hline & Spin eigenfunctions & 52 & 211 & 197 \\
\hline & $\Delta E^{2}$ & 0.0 & 0.22 & 0.67 \\
\hline \multirow{3}{*}{${ }^{1} B_{3 y}$} & Configurations & 96 & 260 & 336 \\
\hline & Spin eigenfunctions & 144 & 504 & 757 \\
\hline & $\Delta E^{2}$ & 0.0 & 0.81 & 1.19 \\
\hline \multirow{3}{*}{${ }^{1} B_{2 u}$} & Configurations & 32 & 124 & 206 \\
\hline & Spin eigenfunctions & 40 & 196 & 562 \\
\hline & $\Delta E^{2}$ & 0.0 & 0.63 & 0.58 \\
\hline
\end{tabular}

${ }^{2} \Delta E$ is the CI energy lowering relative to the GVB CI energy (in eV).

${ }^{b}$ Based on GVB CI among the six $\pi$ orbitals only. 
orbitals; see (5). ${ }^{21}$ Consequently, the correlation errors cancel and accurate $n \pi^{*}$ excitation energies are obtained.

Our calculations indicate that the ${ }^{1,3} B_{3 u}\left(n \pi^{*}\right)$ states give rise to the two lowest vertical excitation energies. However, Hochstrasser and Marzzacco ${ }^{22}$ have observed the presence of a second triple state, assigned as $1^{3} B_{1 u}\left(\pi \pi^{*}\right)$, at $\sim 3.4-3.5 \mathrm{eV}$ in the absorption spectrum of pyrazine. Our calculations place the $1^{3} B_{1 u}\left(\pi \pi^{*}\right)$ vertical excitation energy at $4.11 \mathrm{eV}$. There is experimental and theoretical evidence that the large difference $(\gtrsim 0.5 \mathrm{eV})$ between the adiabatic and vertical excitation energies is real. First, electron and ion impact spectra of pyridine reveal a singlet-triplet transition at $4.1 \mathrm{eV}$ (vertical). ${ }^{23}$ Since the $1^{1} B_{1}\left(n \pi^{*}\right)$ state is not observed in these spectra, we assign the $4.1 \mathrm{eV}$ feature to the $1^{3} A_{1}\left(\pi \pi^{*}\right)$ state. This assignment is supported by the similar intensity distribution of the corresponding ${ }^{3} \pi \pi^{*}$ state $\left(1^{3} B_{1 u}\right)$ in benzene at $3.9 \mathrm{eV}$ in the electron impact spectrum. ${ }^{23 b_{2} 24}$ Intuitive$1 y$, one would not expect the excitation energy of the this ${ }^{3} \pi \pi^{*}$ state to shift greatly in the series benzene, pyridine, pyrazine. Finally, CI calculations on benzene analogous to those employed in this work lead to 3.87 $\mathrm{eV}$ for the $1^{3} B_{1 u}\left(\pi \pi^{*}\right)$ vertical excitation energy in excellent agreement with experiment. ${ }^{25}$

The recent opto-acoustic spectroscopic study of pyrazine by Robin et al. ${ }^{26}$ indicates the presence of triplet states at $\sim 4.2 \mathrm{eV}$ and $\sim 4.5 \mathrm{eV}$. On the basis of our calculations, these features were assigned to the $1^{3} B_{2 \ell}\left(n \pi^{*}\right)$ and $1^{3} A_{u}\left(n \pi^{*}\right)$ states, respectively. ${ }^{26}$ The presence of the $1^{1} A_{u}\left(n \pi^{*}\right)$ state in this region is not evident from the opto-acoustic spectrum. This negative result is not unreasonable, since any effect of the weakly absorbing symmetry-forbidden $1^{1} A_{u}\left(n \pi^{*}\right)$ state will be overshadowed by the presence of the strongly absorbing $1{ }^{1} B_{2 u}\left(\pi \pi^{*}\right)$ state.

The small singlet-triplet splitting $(<0.1 \mathrm{eV})$ for the $1^{1,3} A_{u}$ and $1^{1,3} B_{1 \xi}\left(n \pi^{*}\right)$ states is in marked contrast to that $(>0.7 \mathrm{eV})$ for the $1^{1,3} B_{3 u}$ and $1^{1,3} B_{2 \xi}\left(n \pi^{*}\right)$ states. In the Introduction we stated that the $\pi^{*}$ orbital would adjust to maximize the exchange integral with the unpaired nonbonding orbital in the ${ }^{3} n \pi^{*}$ states and adjust to minimize the exchange integral in the ${ }^{1} n \pi^{*}$ states. The Mulliken populations (Table VI) and plots (Fig. 2) of $\pi^{*}$ orbital from the DZ SCF calculations on the ${ }^{1,3} n \pi^{*}$ states clearly manifest this effect. However, for the $1^{1,3} A_{4}$ and $1{ }^{1,3} B_{1}$ states, the $\pi^{*}$ orbital has $a_{2}$ symmetry (in $C_{2 v}$ ), i.e., a node through the nitrogens. The orbital cannot localize on the excited nitrogen to maximize the favorable exchange interaction in the triplet (nor localize on the para nitrogen to minimize the bad exchange interaction in the singlet). Thus, the very small singlet-triplet splittings of the $1^{1,3} A_{u}$ and $1{ }^{1,3} B_{1 \mathrm{e}}$

TABLE VI. Mulliken populations of the $\pi^{*}$ orbitals.

\begin{tabular}{lllll}
\hline \hline State & Excited N & ortho-C & meta-C & para-N \\
\hline${ }^{3} n \pi^{*}$ & 0.283 & 0.187 & 0.036 & 0.271 \\
${ }^{1} n \pi^{*}$ & 0.075 & 0.130 & 0.041 & 0.583 \\
\hline
\end{tabular}

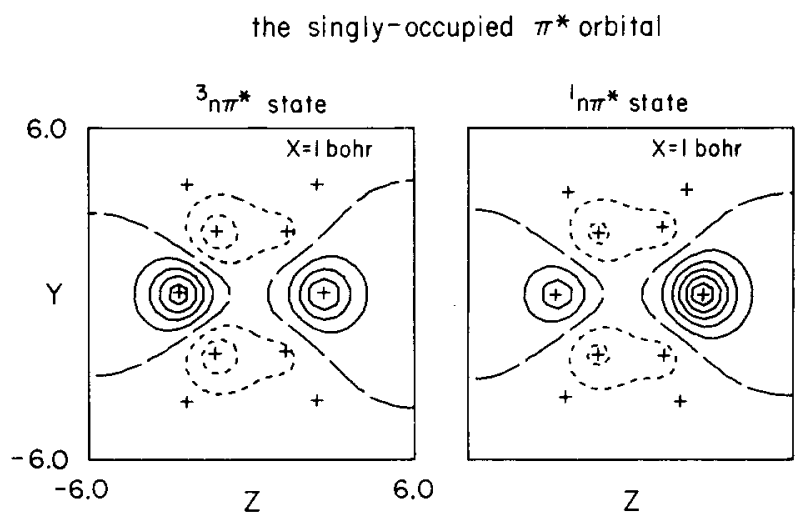

FIG. 2. Contour plots ( $1 a_{0}$ above the molecular plane) of the singly occupied $\pi^{*}$ orbital from the ${ }^{3} n \pi^{*}$ and ${ }^{1} n \pi^{*}$ SCF wavefunctions. The contour increment is $0.05 \mathrm{a}$. $\mathrm{u}$.

\section{states are expected.}

No experimental data are available for the $1^{3} B_{2 u}\left(\pi \pi^{*}\right)$ state at $5.09 \mathrm{eV}$. The agreement $(0.3 \mathrm{eV})$ with optical absorption ${ }^{27}$ and electron impact spectra ${ }^{28}$ for the next state, the $1^{1} B_{2 u}\left(\pi \pi^{*}\right)$, at $5.1 \mathrm{eV}$ is not as good for the $n \pi *$ states. This discrepancy is larger than expected, since comparable CI calculations on benzene give excellent agreement $(<0.1 \mathrm{eV})$ with experiment for the analogous $1^{1} B_{2 u}\left(\pi \pi^{*}\right)$ state. $^{25}$

The location of the "optically forbidden" ${ }^{1} B_{2 \varepsilon}\left(n \pi^{*}\right)$ state has been the subject of much controversy over the past 20 years. Our calculations place the $1{ }^{1} B_{2 g}\left(n \pi^{*}\right)$ at $5.30 \mathrm{eV}$ or $1.30 \mathrm{eV}$ above the "optically allowed" $1^{1} B_{3 u}\left(n \pi^{*}\right)$ state. Numerous direct and indirect spectral measurements have indicated that the $1^{1} B_{2 \xi}$ state is nearly degenerate $(\Delta E<0.1 \mathrm{eV})$ with the $1^{1} B_{3_{u}}$ state. ${ }^{22,29-32}$ However, more detailed spectral analyses $^{33,34}$ in the past and most recent absorption studies on pyrazine $e^{35-39}$ lead to the conclusion that the first singlet-singlet band arises solely from the $1^{1} B_{3 u}$ state without contribution from the $1^{1} B_{2 g}$ state. In fact, a lower limit of $0.8 \mathrm{eV}$ for the $1^{1} B_{3 u}-1^{1} B_{2 \varepsilon}$ splitting energy has been set by Esherick, Zinsli, and El-Sayed ${ }^{40}$ by studying the polarized two-photon phosphorescence excitation spectrum of pyrazine neat crystal at $1.6^{\circ} \mathrm{K}$. Finally, in a beautiful series of simple experiments, Cordes and Moomaw ${ }^{41}$ have actually observed the $1^{1} B_{2 \varepsilon}$ state. Cordes and Moomaw ${ }^{41}$ studied the absorption spectra of pyrazine in a strong hydrogen-bonding solvent (hexafluoroisopropanol/perfluoro- $n$-hexane). By varying the concentration of the alcohol, they are able to hydrogen bond to none, one or both of nitrogen lone pairs in pyrazine. The 1:1 pyrazine-alcohol complex possesses a lower symmetry and the transition to the $1^{1} B_{2 g}\left(2^{1} B_{1}\right.$ in $\left.C_{2 v}\right)$ becomes allowed. This is confirmed by the appearance of a new spectral feature $\sim 1.5 \mathrm{eV}$ above the $1^{1} B_{3 u}\left(1^{1} B_{1}\right.$ in $\left.C_{2 v}\right)$ state. ${ }^{41}$ Adjusting for the large $\left(\sim 2800 \mathrm{~cm}^{-1}\right)$ blue shifts induced by the alcoholic solvent, Cordes and Moomaw ${ }^{41}$ calculate the vertical excitation energy for $1^{1} B_{2 g}$ to be $5.5 \mathrm{eV}$. The good agreement of this result with our calculations confirms the analysis and approach of Cordes and Moomaw, ${ }^{41}$ and reaffirms the correctness of the VB model for lone pair interaction. 
Except for the two $\pi \pi^{*}$ states, $1{ }^{1} B_{1 u}$ and $2{ }^{1} B_{2 u}$, there are no experimental data for comparison with the higher-lying calculated excitation energies. For both ${ }^{1} \pi \pi^{*}$ states, the calculated vertical excitation energies are overestimated by more than $2.0 \mathrm{eV}$ ! Extensive CI calculations by Hay and Shavitt ${ }^{4}$ have shown that the analogous states in benzene have significant ionic character, so that the $\pi$ orbitals are more diffuse than the normal valence excited states. Comparing the CI results in Table IV, the use of the more flexible DZ basis does lead to a $1.0 \mathrm{eV}$ decrease in the ${ }^{1} \pi \pi^{*}$ excitation energies [compare the GVB CI results for the MBS and the $\mathrm{DZ}(\pi)$ POL(3) CI results for the $\mathrm{DZ}$ basis set]. Therefore, we may conclude that the $\mathrm{DZ}$ basis set employed in these calculations is not adequate for describing the ionic $1^{1} B_{1 u}$ and $2{ }^{1} B_{1 u} \pi \pi^{*}$ states. More diffuse $p_{\text {, }}$ basis functions may be required for a proper description. ${ }^{42}$

Finally, our calculations actually allow us to make some semiquantitative statements about the low-lying states of pyridine, for which no ab initio calculations are available. The DZ SCF calculations on the ${ }^{1,3} n \pi^{*}$ states of pyrazine are actually appropriate for pyridine apart from the effect of substituting a nitrogen for the para $\mathrm{CH}$ group. The latter effect is expected to be small $(\sim 0.1 \mathrm{eV})$. As pointed out before, the correlation errors tend to cancel for the $n \pi^{*}$ states, so that the calculated excitation energies should be quite accurate. In fact, comparison of the calculated ${ }^{1} n \pi^{*}$ excitation energy $(4.25 \mathrm{eV})$ to the experimental energy $(4.31 \mathrm{eV})^{27}$ gives good agreement and corroborates our line of reasoning. The experimental information for the lowlying triplet states is sketchy. Electron impact spectra give a firm value of $4.1 \mathrm{eV}$ for the ${ }^{3} A_{1}\left(\pi \pi^{*}\right)$ vertical excitation energy, ${ }^{23}$ and assignments of very weak bands indicate the presence of another(?) triplet state at $\sim 3.7 \mathrm{eV} .{ }^{43.44}$ The DZ SCF calculations lead to 3.63 eV for the ${ }^{3} n \pi^{*}\left({ }^{3} B_{1}\right.$ in pyridine) state excitation energy. The latter result would indicate that the observed transitions ${ }^{43.44}$ near $3.7 \mathrm{eV}$ correspond to ${ }^{3} B_{1}\left(n \pi^{*}\right)$. However, the adiabatic excitation energy for the ${ }^{3} A_{1}\left(\pi \pi^{*}\right)$ state would be expected to be sandwiched between the corresponding ${ }^{3} \pi \pi^{*}$ transitions in benzene $(3.67 \mathrm{eV})^{45}$ and pyrazine $(3.5 \mathrm{eV})^{22}$. Therefore it is not possible to make an assignment solely on the basis of energies.

Analyzing substituent effects, Hoover and Kasha ${ }^{46}$ assigned the lowest triplet state in pyridine to $1^{3} A_{2}\left(n \pi^{*}\right)$. However, our calculations on pyrazine indicate that the $1^{3} A_{2}\left(n \pi^{*}\right)$ state is $\sim 1.5 \mathrm{eV}$ higher than the $1{ }^{3} B_{1}\left(n \pi^{*}\right)$ state by analogy to the splitting of the $1^{3} B_{3 u}$ and $1^{3} A_{u}$ states or $1^{3} B_{2 g}$ and $1^{3} B_{1 g}$ states in pyrazine (cf. Table IV). Disregarding the $1^{3} A_{2}\left(n \pi^{*}\right)$ state (for which there is no concrete experimental evidence), the arguments of Hoover and Kasha ${ }^{46}$ predict the $1^{3} B_{1}\left(n \pi^{*}\right)$ and $1^{3} A_{1}\left(\pi \pi^{*}\right)$ states to be nearly degenerate at $\sim 3.6 \mathrm{eV}$, in agreement with our calculations. Perhaps the near degeneracy of these two states plays a role in the lack of phosphorescence from pyridine.

\section{ACKNOWLEDGMENT}

We thank Professor William R. Moomaw, Dr. Melvin B. Robin, Dr. Peter Esherick, and Professor Mostafa
A. El-Sayed for communicating their experimental results to us prior to publication.

* Contribution No. 5221 from the Arthur Amos Noyes Laboratory of Chemical Physics, California Institute of Technology, Pasadena, CA 91125.

†NSF Predoctoral Fellow, 1970-1973; California Institute Research Foundation Fellow, 1973-1974; Present address: Monsanto Research Corporation, Box 32, Miamisburg, OH 45342 .

\$Partially supported by a Grant (MPS73-05132) from the $\mathrm{Na-}$ tional Science Foundation. Present address: California Instutute of Technology, Pasadena, CA 91125.

§Present address: Theoretical Division, Los Alamos Scientific Laboratory, Los Alamos, NM 87544.

${ }^{1}$ W. R. Wadt and W. A. Goddard III, J. Am. Chem. Soc. 97, 2034 (1975).

${ }^{2}$ (a) R. Hoffmann, A. Imamura, and W. J. Hehre, J. Am. Chem. Soc. 90, 1499 (1968); (b) R. Hoffmann, Acc. Chem. Res. 4, 1 (1971).

${ }^{3} \mathrm{M}$. Hackmeyer and J. L. Whitten, J. Chem. Phys. 54, 3739 (1971).

${ }^{4}$ P. J. Hay and I. Shavitt, Chem. Phys. Lett. 22, 33 (1973); J. Chem. Phys, 60, 2815 (1974).

${ }^{5}$ For convenience, the $\pi$ electrons have been temporarily suppressed. The Mulliken ${ }^{6}$ axis convention is employed in this paper, i. e., the pyrazine molecules lies in the $y z$ plane with the $z$ axis through the nitrogens.

${ }^{6}$ R. S. Mulliken, J. Chem. Phys. 23, 1997 (1955).

${ }^{7}$ P. J. Wheatley, Acta Crystallogr, 10, 182 (1957).

${ }^{8}$ V. S. Schomaker and L. Pauling, J. Am. Chem. Soc. 61, 1769 (1939).

${ }^{9}$ T. H. Dunning, Jr, and P. J. Hay, Modern Theoretical Chemistry, II. Electronic Structure: Ab Initio Methods, edited by H. F. Schaefer. III (Plenum, New York, 1975).

${ }^{10} \mathrm{~T}$. H. Dunning, Jr. and P. J. Hay, Gaussian Easis for Molecular Calculations, Los Alamos Scientific Laboratory Report LA-UR-75-1937.

${ }^{11} \mathrm{~S}$. Huzinaga, J. Chem. Phys. 42, 1293 (1965).

${ }^{12}$ For HF wavefunctions, the doubly occupied orbitals are not uniquely defined by the varational principle. Consequently, the $n$ and $\sigma$ orbitals are often mixed with one another.

${ }^{13}$ W. J. Hunt, W. A. Goddard III, and T. H. Dunning, Jr. , Chem. Phys. Lett. 6, 147 (1970).

${ }^{14}$ Excitations from the $n$ orbitals were not allowed for the $\pi \pi^{*}$ states.

${ }^{15}$ To be consistent for the cations, up to double excitations are allowed from the appropriate $\mathrm{HF}$ cation configuration.

${ }^{16}$ The localizing configuration corresponds to an $n_{+}^{2} n_{-} \rightarrow n_{+} n_{-}^{2}$ or $n_{+} n_{2}^{-} \rightarrow n_{+}^{2} n_{-}$promotion from the dominant configuration. See Ref. 1 for details.

${ }^{17}$ The similarity of the MBS( $\left.\sigma\right)$ POL(1)CI results for the MBS and DZ basis sets is manifest from a comparison of calculations on the $n \pi^{*}$ states in Table IV.

${ }^{18}$ R. Gleiter, E. Heilbronner, and V. Hornung, Angew. Chem. 82, 878 (1970); Helv. Chim. Acta 55, 255 (1972).

${ }^{19}$ N. W. Winter, W. A. Goddard III, and F. W. Bobrowicz, J. Chem. Phys, 62, 4308 (1975).

${ }^{20}$ The decrease in $\pi$ correlation energy when a seventh electron is added may at first seem unreasonable. However, in the seven-electron $\pi$ system, one of the $p_{\text {r }}$ orbitals is doubly occupied (cf. Diagram 4) and hence contributes little to the correlation energy within a six orbital description. Therefore, the seven-electron $\pi$ system with only five singly occupied $p_{\pi}$ orbitals is expected to have a smaller $\pi$ correlation energy than the ground state six-electron $\pi$ system.

${ }^{21} \mathrm{CI}$ calculations have shown for $\mathrm{H}_{2} \mathrm{O}$ that the correlation energies of the in-plane and out-of-plane oxygen lone pairs are indeed comparable. ${ }^{19}$

${ }^{22}$ R. M. Hochstrasser and C. Marzzacco, J. Chem. Phys. 48, 
971 (1968).

${ }^{23}$ (a) J. P. Doering and J. H. Moore, J. Chem. Phys, 56, 2176 (1972); (b) E. H. VanVeen and F. L. Plantenga, Chem. Phys. Lett. 30, 28 (1975).

${ }^{24}$ J. P. Doering, J. Chem. Phys. 51, 2866 (1969).

${ }^{25}$ W. A. Goddard III (unpublished results).

${ }^{26}$ K. Kaya, C. L. Chatelain, M. B. Robin, and N. A. Kuebler, J. Am. Chem. Soc. 97, 2153 (1975).

${ }^{27}$ K. K. Innes, J. P. Byrne, and I. G. Ross, J. Mol. Spectrosc. 22, 125 (1967).

${ }^{28}$ (a) C. Fridh, L. Asbrink, B. O. Jonsson, and E. Lindholm, Int. J. Mass Spectrom. Ion Phys. 8, 101 (1972); (b) M. N. Pisanias, L. S. Christophorou, J. G. Carter, and D. L. McCorkle, J. Chem. Phys. 58, 2110 (1973).

${ }^{29}$ M. Ito, R. Shimada, T. Kuraishi, and W. Mizushima, J. Chem. Phys. 26, 1508 (1957).

${ }^{30}$ (a) M. A. El-Sayed and G. W. Robinson, J. Chem. Phys. 34, 1840 (1961); 35, 1896 (1961); (b) G. W. Robinson and M. A. El-Sayed, Mol. Phys. 4, 273 (1961).

${ }^{31}$ W. R. Moomaw, M. R. DeCamp, and P. C. Podore, Chem. Phys. Lett. 14, 255 (1972).

${ }^{32}$ K. K. Innes, A. H. Kalantar, A. Y. Khan, and T. J. Durnick, J. Mol. Spectrosc. 43, 477 (1972).

${ }^{33}$ K. K. Innes, J. A. Merritt, W. C. Tincher, and S. G. Tilford, Nature (London) 187, 500 (1960).

${ }^{34}$ K. K. Innes, J. D. Simmons, and S. G. Tilford, J. Mol.
Spectrosc. 11, 257 (1963).

${ }^{35}$ C. J. Marzzacco and E. F. Zalewski, J. Mol. Spectrosc. 43, 239 (1972).

${ }^{36}$ H. K. Hong and G. W. Robinson, J. Mol. Spectrose. 52, 1 (1974).

${ }^{37}$ I. Suzuka, N. Mikami, and M. Ito, J. Mol. Spectrosc. 52, 21 (1974).

${ }^{38}$ S. N. Thakur and K. K. Innes, J. Mol. Spectrosc. 52, 130 (1974).

${ }^{39}$ E. F. Zalewski, D. S. McClure, and D. L. Narva, J. Chem. Phys. 61, 2964 (1974).

${ }^{40}$ P. Esherick, P. Zinsli and M. A. El-Sayed, Chem. Phys. 10, 415 (1975).

${ }^{41} \mathrm{~J}, \mathrm{H}_{0}$. Cordes and W. R. Moomaw. (private communication). Thirteenth Symposium on Molecular Structure and Spectroscopy (The Ohio State University, Columbus, OH, 1875).

${ }^{42} \mathrm{~A}$ significant readjustment in the $\sigma$ system can be expected for such ionic states and a good description would likely require simultaneous correlation of the $\sigma$ and $\pi$ systems.

${ }^{43}$ D. F. Evans, J. Chem. Soc. 1957, 3885.

${ }^{44}$ S. Japar and D. A. Ramsay, J. Chem. Phys. 58, 5832 (1973).

${ }^{45}$ S. P. McGlynn, T. Azumi, and M. Kinoshita, Molecular Spectroscopy of the Triplet State (Prentice-Hall, Englewood Cliffs, NJ, 1969).

${ }^{46} \mathrm{R}$. J. Hoover and M. Kasha, J. Am. Chem. Soc. 91, 6508 (1969). 